\title{
Clinical Features and Factors Associated with Occult Gastrointestinal Bleeding in COVID-19 Patients
}

\author{
Xi Zhao* \\ Meihui Tao (ID* \\ Chaoyue Chen (ID \\ Ying Zhang (D) \\ Yu Fu (iD
}

Department of Gastroenterology, Union Hospital, Tongji Medical College, Huazhong University of Science and Technology, Wuhan, People's Republic of China

*These authors contributed equally to this work
Correspondence: Yu Fu

Department of Gastroenterology, Union Hospital, Tongii Medical College,

Huazhong University of Science and

Technology, Wuhan, Hubei, 430022.

People's Republic of China

Tel +8613907/94622

Email futureyu@hust.edu.cn
Background: There has been an increasing number of COVID-19 patients around the world. Since some patients developed with gastrointestinal bleeding, our study focused on the clinical features and gastroscopic findings of these patients, and factors associated with occult gastrointestinal bleeding.

Patients and Methods: In this retrospective, observational study, we collected 368 COVID-19 patients who performed fecal or gastric occult blood from Wuhan Tongji Hospital, Jin Yin-tan Hospital, and Wuhan Union Hospital between February 1, 2020 and March 6, 2020. Clinical features were compared between patients with or without occult gastrointestinal bleeding, and gastroscopic findings of seven patients were described. Logistic regression analyses were performed to explore the factors associated with occult gastrointestinal bleeding.

Results: In total, 43 (11.7\%) patients presented occult gastrointestinal bleeding, whereas 35 $(81.4 \%)$ of severe cases. CRP level, prothrombin time and D-dimer were higher, while lymphocyte count and albumin levels were decreased in patients with occult gastrointestinal bleeding. Gastroscopy in seven COVID-19 patients showed mucosal congestion, erosion or scattered bleeding at different sites. Albumin levels (OR, 0.856 [95\% CI 0.793-0.924]; p < $0.001)$, prothrombin time $(\mathrm{OR}, 1.267$ [1.089-1.475]; $\mathrm{p}=0.002)$ on admission and severe disease (OR, 4.157 [1.765-9.791]; $p=0.001)$ were independent factors associated with GIB in COVID-19 patients, while antiviral drugs and glucocorticoid therapy were not associated with it.

Conclusion: COVID-19 patients with occult gastrointestinal bleeding suffered from worse prognosis. Patients with decreased serum albumin levels or prolonged prothrombin time, and severe cases were at higher risk of occult gastrointestinal bleeding.

Keywords: COVID-19, occult gastrointestinal bleeding, clinical characteristics, related factors

\section{Introduction}

The global pandemic of SARS-CoV-2 has posed a huge challenge to the world. ${ }^{1}$ As of August 23, 2021, the number of confirmed cases worldwide has risen to more than $200,000,000$, with an alarming mortality rate of $2 \% .^{2}$ The typical clinical symptoms of COVID-19 are fever, cough, dyspnea, fatigue and myalgia., Digestive symptoms are in the range of $2 \%$ to $18.6 \%$, including nausea, vomiting, diarrhea, abdominal pain, and the incidence of digestive symptoms was higher in the late epidemic period than early. ${ }^{5}$ Gastrointestinal bleeding (GIB) is in the range of $4 \%$ to $13.7 \%$, which is one of the complications in critically ill patients. ${ }^{6,7}$ Previous studies reported that patients with GIB during the hospitalization had 
a higher mortality risk. ${ }^{8}$ Patients with COVID-19 sometimes received treatments, which could potentially cause damage to the digestive tract, including glucocorticoids, antiviral agents, mechanical ventilation, extracorporeal membrane oxygenation (ECMO) and anticoagulants.

As we know, the angiotensin converting enzyme 2 (ACE2) receptor is also expressed in the epithelial cells of the gastrointestinal, and SARS-CoV-2 binds to ACE2 to exert an effect. ${ }^{9}$ Treatment of GIB in patients with SARS$\mathrm{CoV}-2$ presents unique challenges. Although there are some studies on gastrointestinal bleeding in COVID-19 patients, the direction and specific content of the studies are different from ours. Here, we discuss the clinical features and gastroscopic findings of COVID-19 patients with occult GIB, and factors associated with occult GIB. We identify potential gastrointestinal bleeding patients, which is conducive to the formulation of related diagnosis and treatment plans.

\section{Methods}

\section{Patients and Study Design}

In this multicenter, retrospective study, the subjects included were laboratory-confirmed COVID-19 patients from Wuhan Tongji Hospital (designated hospital for COVID-19), Jin Yin-tan Hospital (designated hospital for COVID-19) and Wuhan Union Hospital main district (non-designated hospital) between February 1, 2020 and March 6, 2020. Throat swab specimens were collected, and SARS-CoV-2 RNA was detected by real-time reverse transcription PCR (rRT-PCR). All patients received a standard treatment based on "Diagnosis and Treatment Protocol for Novel Coronavirus Pneumonia (Trial Version 7)". ${ }^{10}$ Data were extracted for demographic characteristics, laboratory values, comorbidities, complications, treatments, outcomes and gastroscopic findings using electronic medical records.

\section{Definitions}

Laboratory confirmed cases referred to patients who were tested positive for SARS-CoV-2 in throat swab samples. Sepsis and ARDS were diagnosed according to WHO interim guidance and acute kidney injury (AKI) was determined based on KDIGO clinical practice guidelines. ${ }^{11,12}$ Acute cardiac injury was diagnosed according to serum levels of cardiac biomarkers, electrocardiography, and echocardiography. ${ }^{4}$ Acute liver injury was confirmed if abnormalities were seen in alanine aminotransferase
(ALT), aspartate aminotransferase (AST), total bilirubin (TBil), alkaline phosphatase (ALP) or $\gamma$-glutamyl transpeptidase (GGT). The date of symptom onset was defined as the day when initial symptoms were noticed. The baseline values of laboratory examinations were the results on admission, while the extremum values were the maximum or minimum results after hospitalization.

\section{Statistical Analysis}

Continuous variables and categorical variables were expressed as median (interquartile range, IQR) and number $(\%)$, respectively. Independent $t$-test, Mann-Whitney $U, \chi^{2}$ test and Fisher's exact test were used under appropriate conditions. Logistic regression model was adopted to determine the independent factors associated with occult GIB, and adjusted odds ratio (OR) and 95\% confidence interval (CI) were calculated. Given that a total of 43 patients with occult blood tested positive in our study, we enrolled five variables for multivariate logistic regression analysis. Previous studies have shown that gastrointestinal bleeding is more common in critically ill or dead patients, C-reactive protein is a risk factor for severe COVID-19, and coagulation disorder is a risk factor for gastrointestinal bleeding. ${ }^{6,13,14}$ The use of glucocorticoids and antiviral drugs may increase the risk of gastrointestinal bleeding. Therefore, disease severity, antiviral therapy, glucocorticoid therapy, albumin level and prothrombin time were entered into logistic regression models. SPSS (Statistical Package for the Social Sciences) version 22.0 software was applied for all statistical analyses. The results were two-tailed, and $\mathrm{p}$ value $<0.05$ was considered statistically significant.

\section{Results}

\section{Baseline Characteristics, Treatments, and Outcomes}

We initially collected 558 patients with COVID-19, but a total of 368 patients were included in the final analyses after exclusion of those who had not taken fecal or gastric juice occult blood tests. As outlined in Table 1, the median age of all subjects was 57.0 years (IQR 39.0-68.0), and 191 were men. There were $43(11.7 \%)$ cases with occult GIB (38 cases with fecal occult blood test, 5 cases with gastric juice occult blood test) that had a median age of 67.0 years (57.0-71.0), while those without occult GIB were much younger (55.0 years [38.5-67.0], $\mathrm{p}<0.001)$. The percentage of males in patients with occult GIB was 
Table I Baseline Characteristics, Treatments, and Outcomes of Patients with COVID-19 with and without Occult Gastrointestinal Bleeding

\begin{tabular}{|c|c|c|c|c|}
\hline & Total $(\mathrm{N}=368)$ & GIB $(n=43)$ & Non-GIB $(n=325)$ & p value \\
\hline \multicolumn{5}{|l|}{ Demographic characteristics } \\
\hline Age, years & $57.0(39.0-68.0)$ & $67.0(57.0-71.0)$ & $55.0(38.5-67.0)$ & $<0.001$ \\
\hline Male & 191 (5I.9\%) & $30(69.8 \%)$ & I6I (49.5\%) & 0.013 \\
\hline \multicolumn{5}{|l|}{ Comorbidities } \\
\hline Diabetes & $53(14.4 \%)$ & $10(23.3 \%)$ & 43 (I3.2\%) & 0.078 \\
\hline Hypertension & $96(26.1 \%)$ & 17 (39.5\%) & 79 (24.3\%) & 0.033 \\
\hline Cardiovascular disease & $34(9.2 \%)$ & $8(18.6 \%)$ & $26(8.0 \%)$ & 0.043 \\
\hline COPD & II (3.0\%) & $3(7.0 \%)$ & $8(2.5 \%)$ & 0.126 \\
\hline Chronic liver disease & 31 (8.4\%) & 9 (20.9\%) & $22(6.8 \%)$ & 0.005 \\
\hline Chronic kidney disease & $5(1.4 \%)$ & I (2.3\%) & $4(1.2 \%)$ & 0.465 \\
\hline Malignancy & $17(4.6 \%)$ & $6(14.0 \%)$ & II (3.4\%) & 0.008 \\
\hline Immunodeficiency disease & $4(1.1 \%)$ & I (2.3\%) & $3(0.9 \%)$ & 0.393 \\
\hline \multicolumn{5}{|l|}{ Symptoms } \\
\hline Fever & 329 (89.4\%) & $40(93.0 \%)$ & 289 (88.9\%) & 0.598 \\
\hline Chills & $50(13.6 \%)$ & $6(14.0 \%)$ & 44 (13.5\%) & $0.94 I$ \\
\hline Cough & 251 (68.2\%) & 31 (72.1\%) & $220(67.7 \%)$ & 0.560 \\
\hline Chest tightness/chest Pain & 97 (26.4\%) & 9 (20.9\%) & $88(27.1 \%)$ & 0.390 \\
\hline Dyspnea & $59(16.0 \%)$ & $8(18.6 \%)$ & 51 (I5.7\%) & 0.625 \\
\hline Fatigue & 115 (31.3\%) & $10(23.3 \%)$ & $105(32.3 \%)$ & 0.229 \\
\hline Myalgia & $54(14.7 \%)$ & $2(4.7 \%)$ & $52(16.0 \%)$ & 0.048 \\
\hline Anorexia & 161 (43.8\%) & $22(51.2 \%)$ & $139(42.8 \%)$ & 0.297 \\
\hline Diarrhea & 101 (27.4\%) & $8(18.6 \%)$ & $93(28.6 \%)$ & 0.167 \\
\hline Nausea or vomiting & 29 (7.9\%) & $6(14.0 \%)$ & $23(7.1 \%)$ & 0.130 \\
\hline Other & $122(33.2 \%)$ & $14(32.6 \%)$ & $108(33.2 \%)$ & 0.930 \\
\hline \multicolumn{5}{|l|}{ Disease severity status } \\
\hline Non-severe & $221(60.1 \%)$ & $8(18.6 \%)$ & $213(65.5 \%)$ & \\
\hline Severe & 147 (39.9\%) & $35(81.4 \%)$ & $112(34.5 \%)$ & $<0.001$ \\
\hline \multicolumn{5}{|l|}{ Complications } \\
\hline Sepsis & $10(2.7 \%)$ & 5 (I I.6\%) & $5(1.5 \%)$ & 0.003 \\
\hline ARDS & $40(10.9 \%)$ & $17(39.5 \%)$ & $23(7.1 \%)$ & $<0.001$ \\
\hline Acute liver injury & 146 (39.7\%) & $28(65.1 \%)$ & 118 (36.3\%) & $<0.001$ \\
\hline Acute kidney injury & $54(14.7 \%)$ & $16(37.2 \%)$ & 38 (11.7\%) & $<0.001$ \\
\hline Acute cardiac injury & $34(9.2 \%)$ & $20(46.5 \%)$ & 14 (4.3\%) & $<0.001$ \\
\hline Coagulopathy & $48(13.0 \%)$ & $20(46.5 \%)$ & $28(8.6 \%)$ & $<0.001$ \\
\hline Hypotension & $24(6.5 \%)$ & $10(23.3 \%)$ & 14 (4.3\%) & $<0.001$ \\
\hline \multicolumn{5}{|l|}{ Treatments } \\
\hline Mechanical ventilation & $63(17.1 \%)$ & 32 (74.4\%) & 31 (9.5\%) & $<0.001$ \\
\hline Antibiotic therapy & 332 (90.2\%) & $4 \mathrm{I}(95.3 \%)$ & 291 (89.5\%) & 0.286 \\
\hline Antiviral therapy & 355 (96.5\%) & $37(86.0 \%)$ & $318(97.8 \%)$ & 0.002 \\
\hline Glucocorticoid therapy & $153(41.6 \%)$ & $32(74.4 \%)$ & $12 \mid(37.2 \%)$ & $<0.001$ \\
\hline \multicolumn{5}{|l|}{ Outcomes } \\
\hline ICU & 37 (10.1\%) & $23(53.5 \%)$ & $14(4.3 \%)$ & $<0.001$ \\
\hline Death & 65 (I7.7\%) & $32(74.4 \%)$ & $33(10.2 \%)$ & $<0.001$ \\
\hline
\end{tabular}

Note: Data are presented as median (interquartile range) or as $\mathrm{n}(\%)$.

Abbreviations: COVID-19, coronavirus disease 2019; COPD, Chronic obstructive pulmonary disease; ARDS, acute respiratory distress syndrome. 
significantly higher relative to those without occult GIB (69.8\% vs $49.5 \%, p=0.013)$. Pre-existing comorbidities were more common in occult GIB group, though only hypertension (17 [39.5\%]), cardiovascular disease (8 [18.6\%]), chronic liver disease (9 [20.9\%]) and malignancy $(6[14.0 \%])$ showed significant difference between two groups. The most common symptoms were fever (40 [93.0\%]), followed by cough (31 [72.1\%]), anorexia (22 $[51.2 \%])$, fatigue (10 [23.3\%]), and nausea or vomiting (6 [14.0\%]). Except for myalgia $(\mathrm{p}=0.048)$, the difference in clinical symptoms between the two groups was not statistically significant.

Compared to patients without occult GIB, severe cases accounted for a larger proportion in occult GIB group ( $34.5 \%$ vs $81.4 \%, \mathrm{p}<0.001)$ and were prone to other complications, including sepsis ( $1.5 \%$ vs $11.6 \%, \mathrm{p}=0.003)$, ARDS $(7.1 \%$ vs $39.5 \%, \mathrm{p}<0.001)$, acute liver injury (36.3\% vs $65.1 \%, \mathrm{p}<0.001)$, acute kidney injury $(11.7 \%$ vs $37.2 \%, p<0.001)$, acute cardiac injury $(4.3 \%$ vs $46.5 \%$, $\mathrm{p}<0.001)$ and coagulopathy $(8.6 \%$ vs $46.5 \%, \mathrm{p}<0.001)$. The proportions of patients who received glucocorticoid therapy and mechanical ventilation were higher $(74.4 \%$ vs $37.2 \%, \mathrm{p}<0.001$ and $74.4 \%$ vs $9.5 \%, \mathrm{p}<0.001$, respectively), while antiviral therapy accounted for a lower proportion $(86.0 \%$ vs $97.8 \%, \mathrm{p}=0.002)$. The mortality rate was significantly higher in patients with occult GIB $(74.4 \%$ vs $10.2 \%, \mathrm{p}<0.001)$ where 32 out of 43 patients were dead. Among the deceased patients with occult GIB, the median time from symptoms onset to death was 19.0 days (18.0-24.8), and the median time from positive occult blood tests to death was 5.0 days (2.0-9.0).

We performed gastroscopy on seven COVID-19 patients (Figure 1). Gastric mucosal lesions were mainly manifested as congestion, erosion or scattered bleeding, while esophageal mucosa, pylorus and duodenum were normal.

\section{Laboratory Findings}

Baseline laboratory values are shown in Table 2. Patients with occult GIB showed higher levels of C-reactive protein (CRP), white blood count, AST, TBil, lactate dehydrogenase (LDH), creatine kinase, creatinine, prothrombin time (PT) and D-dimer. However, lymphocyte count, platelet and albumin levels decreased compared with those in the patients without occult GIB. Proportions of hypoalbuminemia and prolonged PT in occult GIB group were significantly higher than those in the patients without occult GIB ( $79.1 \%$ vs $37.0 \%, \mathrm{p}<0.001$ and $23.8 \%$ vs
$2.2 \%, \mathrm{p}<0.001$, respectively). For extremum laboratory values during the disease course (Table 2), statistically significant indicators were similar to those in baseline laboratory values, whereas patients with occult GIB showed higher levels of ALP $(p<0.001)$, GGT $(p=0.003)$ and activated partial thromboplastin time $(\mathrm{p}<0.001)$, and decreased hemoglobin $(\mathrm{p}=0.008)$. With the progress of disease, although the number of patients with hypoalbuminemia or prolonged PT increased in both groups, the proportion of hypoalbuminemia and the proportion of prolonged PT was always significantly higher in patients with occult GIB (90.7\% vs $51.1 \%$, p $<0.001$ and $51.2 \%$ vs $8.1 \%$, $\mathrm{p}<0.001$, respectively).

\section{Factors Associated with Occult Gastrointestinal Bleeding}

As shown in Table 3, when analyzed by univariable analysis, age, sex, disease severity, hypertension, cardiovascular disease, chronic liver disease, malignancy, CRP, white blood cell count, lymphocyte count, platelet, AST, albumin, TBil, LDH, creatine kinase, creatinine, PT, D-dimer, antiviral therapy, and glucocorticoid therapy were associated with occult GIB. However, in the multivariable logistic regression model, albumin levels (OR, 0.856 [95\% CI 0.793-0.924]; $\mathrm{p}<0.001$ ), prothrombin time (OR, 1.267 [1.089-1.475]; $\mathrm{p}=0.002)$ on admission and severe disease (OR, 4.157 [1.765-9.791]; $\mathrm{p}=0.001$ ) were independent factors associated with occult GIB in COVID-19 patients.

\section{Discussion}

To our knowledge, this is the first retrospective cohort study to discuss the clinical features of patients with occult gastrointestinal bleeding and factors associated with it. In particular, severe cases, decreased serum albumin levels and prolonged PT on admission were associated with occult gastrointestinal bleeding. Of all subjects, $43(11.7 \%)$ had occult gastrointestinal bleeding, while Yang et al found only $2(4 \%)$ cases with GIB in their study. ${ }^{6}$ This may be related to the fact that we enrolled patients with fecal or gastric juice occult blood test and the relatively large sample size. A majority of patients performed occult blood tests because of diarrhea, abdominal discomfort, or anemia.

In our study, we found that the baseline level of albumin was an independent related factor for occult GIB, and $90.7 \%$ patients with occult GIB suffered from 



Figure I Gastroscopy of seven patients with SARS-CoV-2 infection. The images showed different parts of the stomach under the endoscopy. (A-E) Scattered bleeding and congestion of the gastric fundus. (F-M) Scattered bleeding of the gastric body. ( $\mathbf{N}$ and $\mathbf{O})$ Scattered bleeding of the antrum. 
Table 2 Laboratory Findings of Patients with COVID-19 with and without Occult Gastrointestinal Bleeding

\begin{tabular}{|c|c|c|c|c|}
\hline Baseline & Total $(\mathbf{N}=\mathbf{3 6 8})$ & GIB $(n=43)$ & Non-GIB $(n=325)$ & $\mathrm{p}$ value \\
\hline CRP, mg/L & $16.8(3.1-57.3)$ & $79.3(33.2-151.6)$ & $12.8(3.1-48.5)$ & $<0.001$ \\
\hline White blood cell count, $\times 10^{9} / \mathrm{L}$ & $5.1(3.9-6.9)$ & $7.8(4.4-15.6)$ & $5.0(3.8-6.5)$ & $<0.001$ \\
\hline Lymphocyte count, $\times 10^{9} / \mathrm{L}$ & I.I (0.7-1.5) & $0.6(0.4-0.7)$ & $1.2(0.8-1.6)$ & $<0.001$ \\
\hline Platelet count, $\times 10^{9} / \mathrm{L}$ & $195.0(147.0-256.3)$ & $161.0(113.0-238.0)$ & $197.0(151.0-265.0)$ & 0.001 \\
\hline Hemoglobin, g/L & $129.0(\mid 17.0-138.0)$ & $126.0(117.0-144.0)$ & $129.0(\mid 17.0-137.0)$ & 0.839 \\
\hline$<60$ & I ( $(0.3 \%)$ & I (2.3\%) & $0(0.0 \%)$ & 0.117 \\
\hline ALT, U/L & $21.0(15.0-34.0)$ & $26.0(16.0-42.0)$ & $21.0(15.0-33.0)$ & 0.158 \\
\hline AST, U/L & $26.0(19.0-37.8)$ & $42.0(29.0-59.0)$ & $25.0(19.0-35.0)$ & $<0.001$ \\
\hline Albumin, $g / L$ & $36.2(31.7-40.0)$ & $30.9(28.7-33.6)$ & $36.9(32.6-40.2)$ & $<0.001$ \\
\hline$<35$ & 153/365 (4I.9\%) & $34 / 43(79.1 \%)$ & $119 / 322(37.0 \%)$ & $<0.001$ \\
\hline Total bilirubin, $\mu \mathrm{mol} / \mathrm{L}$ & $9.3(6.8-12.6)$ & $14.0(9.3-19.0)$ & $8.9(6.6-11.9)$ & $<0.001$ \\
\hline ALP, U/L & $65.0(53.3-79.0)$ & $66.0(55.0-88.0)$ & $65.0(53.0-78.0)$ & 0.197 \\
\hline GGT, U/L & $24.0(16.0-46.8)$ & $28.0(19.0-54.0)$ & $24.0(16.0-45.0)$ & 0.214 \\
\hline $\mathrm{LDH}, \mathrm{U} / \mathrm{L}$ & $247.0(194.0-336.0)$ & $480.0(284.0-655.0)$ & $233.5(187.5-307.8)$ & $<0.001$ \\
\hline Creatine kinase, U/L & $68.0(48.0-129.8)$ & $106.0(56.0-246.0)$ & $67.0(48.0-120.0)$ & 0.013 \\
\hline Creatinine, $\mu \mathrm{mol} / \mathrm{L}$ & $69.2(58.0-84.0)$ & $80.0(63.0-96.0)$ & $69.0(57.9-82.3)$ & 0.005 \\
\hline PT, s & $13.3(12.4-14.1)$ & $14.2(12.9-15.7)$ & $13.3(12.3-14.0)$ & $<0.001$ \\
\hline$\geq 16$ & $17 / 359(4.7 \%)$ & 10/42 (23.8\%) & $7 / 317$ (2.2\%) & $<0.001$ \\
\hline APTT, s & $36.6(30.9-40.8)$ & $37.9(31.8-41.8)$ & $36.4(30.5-40.8)$ & 0.094 \\
\hline D-dimer, ug/mL & $0.6(0.3-1.4)$ & $2.1(0.9-11.4)$ & $0.5(0.3-1.2)$ & $<0.001$ \\
\hline \multicolumn{5}{|l|}{ Extremum } \\
\hline CRP, mg/L & $21.1(3.6-64.6)$ & $157.3(59.5-200.3)$ & $16.8(3.2-51.6)$ & $<0.001$ \\
\hline White blood cell count, $\times 10^{9} / \mathrm{L}$ & $6.8(5.2-9.6)$ & $15.6(7.7-24.8)$ & $6.5(5.0-8.5)$ & $<0.001$ \\
\hline Lymphocyte count, $\times 10^{9} / \mathrm{L}$ & $1.0(0.6-1.4)$ & $0.4(0.2-0.6)$ & $1.0(0.7-1.5)$ & $<0.001$ \\
\hline Platelet count, $\times 10^{9} / \mathrm{L}$ & $246.0(187.0-316.0)$ & $176.0(87.0-273.0)$ & $248.0(197.0-327.0)$ & $<0.001$ \\
\hline Hemoglobin, g/L & $122.0(109.0-132.0)$ & II7.0 (87.0-129.0) & $122.0(|1| 1.0-132.0)$ & 0.008 \\
\hline$<60$ & $\mathrm{I}(0.3 \%)$ & I (2.3\%) & $0(0.0 \%)$ & 0.117 \\
\hline ALT, U/L & $32.0(20.0-57.0)$ & $41.0(21.0-74.0)$ & $32.0(19.5-51.0)$ & 0.072 \\
\hline AST, U/L & $31.0(21.0-46.0)$ & $52.0(36.0-84.0)$ & $29.0(21.0-41.5)$ & $<0.001$ \\
\hline Albumin, $g / L$ & $33.7(29.9-38.0)$ & $26.8(22.8-31.7)$ & $34.8(30.8-38.3)$ & $<0.001$ \\
\hline$<35$ & $205(55.7 \%)$ & 39 (90.7\%) & $166(51.1 \%)$ & $<0.001$ \\
\hline Total bilirubin, $\mu \mathrm{mol} / \mathrm{L}$ & II.5 (8.5-15.9) & $17.7(13.5-35.9)$ & $11.1(8.2-14.8)$ & $<0.001$ \\
\hline ALP, U/L & $74.0(60.0-94.8)$ & $94.0(74.0-142.0)$ & $71.0(58.0-88.0)$ & $<0.001$ \\
\hline GGT, U/L & $32.0(19.0-58.0)$ & $48.0(27.0-96.0)$ & $31.0(19.0-57.0)$ & 0.003 \\
\hline $\mathrm{LDH}, \mathrm{U} / \mathrm{L}$ & $262.5(200.8-373.5)$ & $651.0(417.0-1093.0)$ & $249.0(197.0-326.0)$ & $<0.001$ \\
\hline
\end{tabular}

(Continued) 
Table 2 (Continued).

\begin{tabular}{|l|l|l|l|l|}
\hline Baseline & Total $(\mathbf{N}=\mathbf{3 6 8})$ & GIB $(\mathbf{n = 4 3 )}$ & Non-GIB (n=325) & P value \\
\hline Creatine kinase, U/L & $72.0(49.8-146.3)$ & $163.0(67.0-495.5)$ & $67.0(48.0-129.3)$ & $<0.001$ \\
\hline Creatinine, $\mu \mathrm{mol} / \mathrm{L}$ & $75.0(61.0-92.0)$ & $96.0(76.0-175.0)$ & $73.0(61.0-87.6)$ & $<0.001$ \\
\hline PT, s & $13.6(12.8-14.5)$ & $16.0(13.6-20.3)$ & $13.4(12.5-14.3)$ & $<0.001$ \\
\hline$\geq 16$ & $48 / 363(13.2 \%)$ & $22 / 43(51.2 \%)$ & $26 / 320(8.1 \%)$ & $<0.001$ \\
\hline APTT, s & $37.8(33.1-42.9)$ & $44.8(40.0-56.6)$ & $37.1(32.4-42.0)$ & $<0.001$ \\
\hline D-dimer, ug/mL & $0.7(0.3-2.2)$ & $16.2(1.7-21.0)$ & $0.6(0.3-1.5)$ & $<0.001$ \\
\hline
\end{tabular}

Notes: Data are presented as median (interquartile range) or as $\mathrm{n}(\%)$. Baseline laboratory findings were the results on admission, while the extremum were the maximum or minimum results after hospitalization.

Abbreviations: COVID-19, coronavirus disease 2019; CRP, C-reactive protein; ALT, alanine aminotransferase; AST, aspartate aminotransferase; ALP, alkaline phosphatase; GGT, $\gamma$-glutamyl transpeptidase; LDH, lactate dehydrogenase; PT, prothrombin time; APTT, activated partial thromboplastin time.

hypoalbuminemia throughout the course of the disease. Serum albumin is one of the most important proteins in human plasma, the function of which is to maintain stabilization of blood colloid osmotic pressure and nutrition. In animal experiments, it was proved that serum albumin has protective effects, including improving arterial hyporeactivity in patients with endotoxemia, reducing ischemia-reperfusion injury, and exerting antiinflammatory effects. ${ }^{15-17}$ Hypoalbuminemia is associated with poor prognosis in many diseases and patients with hypoalbuminemia had higher mortality rate and longer length of hospital stay. ${ }^{18}$ Low serum albumin levels may cause mucosal edema through decreasing the plasma colloid osmotic pressure, which may exacerbate mucosal damage caused by stress or other factors. Previous study has demonstrated that serum albumin is a significant predictor of short-term mortality in patients with upper gastrointestinal bleeding, and intravenous infusion of albumin shortens the duration of hospitalization for patients with peptic ulcer bleeding complicated with hypoalbuminemia. ${ }^{19}$

Based on our results, the risk for occult gastrointestinal bleeding was much higher in severe patients, which was possibly because of stress-related mucosal disease (SRMD) in critical cases. SRMD may be caused by hypotension, hypovolaemia, high levels of catecholamines, release of proinflammatory cytokine or vasoconstriction. ${ }^{20}$ In our study, seven critically ill patients showed SRMD under gastroscopy. In intensive care unit, the frequency of clinically important bleeding from SRMD was $2.6 \%$. For patients with risk factors for SRMD bleeding, including mechanical ventilation for over 48 hours and coagulopathy, stress-ulcer prophylaxis (SUP) can be used to inhibit gastric acid secretion. ${ }^{21}$ Previous study reported that $2(4 \%)$ patients with GIB all died. ${ }^{6}$ Our results showed that patients with occult GIB had higher mortality rate (32 [74.4\%]), and the median time from positive occult blood test to death was 5.0 days. Moreover, the proportions of organ injury and mechanical ventilation were remarkably higher in cases with occult GIB. All these results indicated that patients with occult GIB had a poor prognosis.

Coagulation disorder can be observed in severe infections, sepsis, septic shock and COVID-19, some of whom present prolonged PT. ${ }^{22,23}$ In our study, PT was another factor associated with occult gastrointestinal bleeding, and every 1s increase in PT will increase the risk of occult GIB by 1.267 times. Previously, coagulopathy has been reported as a risk factor for gastrointestinal bleeding, prolonged PT may aggravate gastrointestinal bleeding caused by mucosal damage. ${ }^{14}$ Inflammatory response and coagulation are two important host defense mechanisms, and the response will increase with the severity of the disease, which may cause potential damage to the host. ${ }^{24}$ Coagulation abnormality is associated with an increased risk of death in patients with COVID-19. Autopsy results of COVID-19 patients show typical platelet-rich thrombus deposits in the small vessels of the lungs and other organs. It suggests that coagulopathy associated with COVID-19 is the combination of low-grade disseminated intravascular coagulation and localised pulmonary thrombotic microangiopathy, which may have a significant impact on organ dysfunction. ${ }^{25}$ In critically ill patients, cytokine storm characterized by high concentrations of proinflammatory cytokines and chemokines can be observed, and the release of tumor necrosis factor (TNF- 
Table 3 Multivariable Logistic Regression Identifying Independent Factors Associated with Occult Gastrointestinal Bleeding

\begin{tabular}{|c|c|c|c|c|}
\hline & \multirow{2}{*}{$\begin{array}{l}\text { Univariable Analysis } \\
\text { Odds Ratio }(95 \% \mathrm{CI})\end{array}$} & \multirow[t]{2}{*}{ p value } & \multirow{2}{*}{$\begin{array}{l}\text { Multivariable Analysis } \\
\text { Odds Ratio }(95 \% \mathrm{CI})\end{array}$} & \multirow[t]{2}{*}{$\mathrm{p}$ value } \\
\hline & & & & \\
\hline \multicolumn{5}{|l|}{ Baseline characteristics } \\
\hline Age, years & $1.043(1.020-1.067)$ & $<0.001$ & & \\
\hline Male & $2.351(1.184-4.669)$ & 0.015 & & \\
\hline Severe & $8.320(3.733-18.543)$ & $<0.001$ & $4.157(1.765-9.791)$ & 0.001 \\
\hline \multicolumn{5}{|l|}{ Comorbidities } \\
\hline Diabetes & $1.987(0.914-4.322)$ & 0.083 & & \\
\hline Hypertension & $1.050(2.036-3.946)$ & 0.035 & & \\
\hline Cardiovascular disease & $2.629(1.105-6.25 I)$ & 0.029 & & \\
\hline COPD & $2.972(0.757-|| 1.66 \mid)$ & 0.118 & & \\
\hline Chronic liver disease & $3.646(1.554-8.553)$ & 0.003 & & \\
\hline Chronic kidney disease & $1.911(0.209-17.501)$ & 0.567 & & \\
\hline Malignancy & $4.629(1.618-13.247)$ & 0.004 & & \\
\hline Immunodeficiency disease & $2.556(0.260-25.133)$ & 0.421 & & \\
\hline \multicolumn{5}{|l|}{ Baseline laboratory findings } \\
\hline CRP, mg/L & $1.016(1.011-1.022)$ & $<0.001$ & & \\
\hline White blood cell count, $\times 10^{9} / \mathrm{L}$ & $1.300(1.191-1.419)$ & $<0.001$ & & \\
\hline Lymphocyte count, $\times 10^{9} / \mathrm{L}$ & $0.109(0.046-0.257)$ & $<0.001$ & & \\
\hline Platelet count, $\times 10^{9} / \mathrm{L}$ & $0.991(0.986-0.996)$ & 0.001 & & \\
\hline Hemoglobin, g/L & $1.000(0.984-1.017)$ & 0.991 & & \\
\hline ALT, U/L & $1.005(0.995-1.014)$ & 0.313 & & \\
\hline AST, U/L & $1.023(1.012-1.035)$ & $<0.001$ & & \\
\hline Albumin, $g / L$ & $0.824(0.767-0.885)$ & $<0.001$ & $0.856(0.793-0.924)$ & $<0.001$ \\
\hline Total bilirubin, $\mu \mathrm{mol} / \mathrm{L}$ & $1.093(1.045-1.143)$ & $<0.001$ & & \\
\hline ALP, U/L & 1.007 (1.000-1.015) & 0.054 & & \\
\hline GGT, U/L & 1.001 (0.998-I.005) & 0.509 & & \\
\hline $\mathrm{LDH}, \mathrm{U} / \mathrm{L}$ & $1.004(1.003-1.006)$ & $<0.001$ & & \\
\hline Creatine kinase, U/L & $1.002(1.001-1.003)$ & 0.002 & & \\
\hline Creatinine, $\mu \mathrm{mol} / \mathrm{L}$ & $1.016(1.006-1.025)$ & 0.001 & & \\
\hline PT, s & $1.423(1.201-1.685)$ & $<0.001$ & I.267 (I.089-I.475) & 0.002 \\
\hline APTT, s & $1.038(0.993-1.086)$ & 0.095 & & \\
\hline D-dimer, ug/mL & I.068 (I.027-I.III) & 0.001 & & \\
\hline \multicolumn{5}{|l|}{ Treatments } \\
\hline Antiviral therapy & $0.136(0.043-0.425)$ & 0.001 & $0.416(0.104-1.666)$ & 0.215 \\
\hline Glucocorticoid therapy & $4.905(2.385-10.086)$ & $<0.001$ & I.553 (0.5 I 2-4.707) & 0.437 \\
\hline
\end{tabular}

Note: Baseline laboratory findings were the results on admission.

Abbreviations: $\mathrm{Cl}$, confidence interval; COPD, Chronic obstructive pulmonary disease; CRP, C-reactive protein; ALT, alanine aminotransferase; AST, aspartate aminotransferase; ALP, alkaline phosphatase; GGT, $\gamma$-glutamyl transpeptidase; LDH, lactate dehydrogenase; PT, prothrombin time; APTT, activated partial thromboplastin time.

$\alpha)$ and interleukin can affect coagulation function. ${ }^{26}$ It was demonstrated in a study of rheumatoid arthritis that the use of glucocorticoids may cause gastric mucosal damage. ${ }^{27}$ Clinically, antiviral therapy may induce gastrointestinal adverse effects, including nausea, vomiting, anorexia, diarrhea, abdominal pain, and rare gastrointestinal bleeding. However, our results did not support glucocorticoid and antiviral drugs correlation with gastrointestinal bleeding in COVID-19 patients.
Among the patients with occult GIB, there were only 3 (7.0\%) developed with moderate anemia and one patient had severe anemia. Besides, hematemesis and melena were not found in all participants, and there was no significant difference in the baseline level of hemoglobin and the proportion of severe anemia between patients with or without occult GIB. Thus, there was only minimal hemorrhage in the gastrointestinal tract in COVID-19 patients. Therefore, we consider that gastrointestinal mucosal damage is caused by stress gastric 
mucosal microcirculation disorder or hypoalbuminemia induced mucosal repair dysfunction.

This study has several limitations. First, patients with COVID-19 were unable to perform comprehensive gastrointestinal examination to clarify gastrointestinal mucosal damage and bleeding owing to the restriction of clinical conditions. Second, due to the limitation of retrospective study design, the time of occult blood tested positive may be affected by discontinuous detection of gastrointestinal bleeding. Third, this is a retrospective study that relies on GIB documentation provided by healthcare providers; therefore, some GIB cases may have been missed. The number of patients with GI bleed is small in our study, and we suggest that larger studies need to be carried out in future. Fourth, SARS-CoV-2 was not detected in stool samples; therefore, it is hard to assess the relationship between viral loads and gastrointestinal bleeding.

In general, we found that severe cases, albumin and PT upon admission were independent related factors for occult gastrointestinal bleeding in COVID-19 patients. This may help physicians identify patients with GIB and take enteral nutrition or histamine-2 receptor blockers or proton-pump inhibitors treatment in the early stage. Mucosa erosion in gastrics was observed under gastroscopy. Although SARS-CoV-2 may not cause massive hemorrhage in the gastrointestinal tract, it is worth noting that patients with gastrointestinal bleeding still had worse prognosis.

\section{Ethics Approval}

The study received approval from the ethics commission of Wuhan Tongji Hospital, Wuhan Union Hospital and Jin Yin-tan Hospital (S2020-055, S2020-056, 2020-YJ-047), and was conducted in accordance with the Declaration of Helsinki. Due to the specific nature of the disease, the requirement of informed consent was waived, and all data were anonymized to maintain participants' privacy.

\section{Acknowledgment}

This study is funded by Natural Science Foundation of China (grant number 82070572, 81770554, 81570501). We acknowledge that all of the medical workers participated in the diagnosis and treatment of patients in Wuhan, and the patients involved in this study.

\section{Author Contributions}

All authors made substantial contributions to the work reported, whether that is in the conception, study design, execution, acquisition of data, analysis and interpretation, or in all these areas; took part in drafting, revising or critically reviewing for important intellectual content; have agreed on the journal to which the article will be submitted; gave final approval of the version to be published; agree to take responsibility and be accountable for the contents of the article.

\section{Disclosure}

None of the authors have declared any potential conflicts of interest.

\section{References}

1. World Health Organization. Director-General's opening remarks at the media briefing on COVID-19-11 March 2020. Available from: https://www.who.int/dg/speeches/detail/who-director-general-s-open ing-remarks-at-the-media-briefing-on-covid-19-11-march-2020.

Accessed January 29, 2021.

2. World Health Organization. Coronavirus disease (COVID-19) outbreak situation. Available from: https://www.who.int/emergencies/dis eases/novel-coronavirus-2019. Accessed August 23, 2021.

3. Chen N, Zhou M, Dong X, et al. Epidemiological and clinical characteristics of 99 cases of 2019 novel coronavirus pneumonia in Wuhan, China: a descriptive study. Lancet. 2020;395(10223):507-513. doi:10.1016/S0140-6736(20)30211-7

4. Huang C, Wang Y, Li X, et al. Clinical features of patients infected with 2019 novel coronavirus in Wuhan, China. Lancet. 2020;395 (10223):497-506. doi:10.1016/S0140-6736(20)30183-5

5. Gulen M, Satar S. Uncommon presentation of COVID-19: gastrointestinal bleeding. Clin Res Hepatol Gastroenterol. 2020;44(4):e72e76. doi:10.1016/j.clinre.2020.05.001

6. Yang X, Yu Y, Xu J, et al. Clinical course and outcomes of critically ill patients with SARS-CoV-2 pneumonia in Wuhan, China: a single-centered, retrospective, observational study. Lancet Respir Med. 2020;8(5):475-481. doi:10.1016/S2213-2600(20)30079-5

7. Tian Y, Rong L, Nian W, et al. Review article: gastrointestinal features in COVID-19 and the possibility of faecal transmission. Aliment Pharmacol Ther. 2020;51(9):843-851. doi:10.1111/ apt. 15731

8. Trindade AJ, Izard S, Coppa K, et al. Gastrointestinal bleeding in hospitalized COVID-19 patients: a propensity score matched cohort study. J Intern Med. 2021;289(6):887-894. doi:10.1111/joim.13232

9. Gu J, Han B, Wang J. COVID-19: gastrointestinal manifestations and potential fecal-oral transmission. Gastroenterology. 2020;158 (6):1518-1519. doi:10.1053/j.gastro.2020.02.054

10. Commission CNH. Chinese clinical guidance for COVID-19 pneumonia diagnosis and treatment. Available from: http://kjfy.meeting china.org/msite/news/show/cn/3337.html. Accessed March 4, 2020.

11. Khwaja A. KDIGO clinical practice guidelines for acute kidney injury. Nephron Clin Pract. 2012;120(4):c179-c184. doi:10.1159/ 000339789

12. World Health Organization. Clinical management of severe acute respiratory infection when novel coronavirus (2019-nCoV) infection is suspected-13 March 2020. Available from: https://www.who.int/ publications-detail/clinical-management-of-severe-acute-respiratoryinfection-when-novel-coronavirus-(ncov)-infection-is-suspected. Accessed April 25, 2020.

13. Lu J, Hu S, Fan R, et al. ACP risk grade: a simple mortality index for patients with confirmed or suspected severe acute respiratory syndrome coronavirus 2 disease (COVID-19) during the early stage of outbreak in Wuhan, China. medRxiv. 2020. doi:10.1101/2020.02.2 0.20025510 
14. Cook DJ, Fuller HD, Guyatt GH, et al. Risk factors for gastrointestinal bleeding in critically ill patients. $N$ Engl $J$ Med. 1994;330 (6):377-381. doi:10.1056/NEJM199402103300601

15. Meziani F, Kremer H, Tesse A, et al. Human serum albumin improves arterial dysfunction during early resuscitation in mouse endotoxic model via reduced oxidative and nitrosative stresses. $\mathrm{Am}$ J Pathol. 2007;171(6):1753-1761. doi:10.2353/ajpath.2007.070316

16. Lang JD, Figueroa M, Chumley P, et al. Albumin and hydroxyethyl starch modulate oxidative inflammatory injury to vascular endothelium. Anesthesiology. 2004;100(1):51-58. doi:10.1097/ 00000542-200401000-00012

17. Lee JH, Kim J, Kim K, et al. Albumin and C-reactive protein have prognostic significance in patients with community-acquired pneumonia. J Crit Care. 2011;26(3):287-294. doi:10.1016/j.jcrc.2010.10.007

18. Franch-Arcas G. The meaning of hypoalbuminaemia in clinical practice. Clin Nutr. 2001;20(3):265-269. doi:10.1054/clnu.2001.0438

19. Cheng HC, Chang WL, Chen WY, et al. Intravenous albumin shortens the duration of hospitalization for patients with hypoalbuminemia and bleeding peptic ulcers: a pilot study. Dig Dis Sci. 2013;58 (11):3232-3241. doi:10.1007/s10620-013-2821-8

20. Bardou M, Quenot JP, Barkun A. Stress-related mucosal disease in the critically ill patient. Nat Rev Gastroenterol Hepatol. 2015;12 (2):98-107. doi:10.1038/nrgastro.2014.235

21. Garcia-Rayado G, Lanas A. Upper gastrointestinal bleeding in critically ill patients: proton-pump inhibitors, histamine- 2 receptor antagonists or placebo? Many questions remain unanswered. Curr Med Res Opin. 2018;34(11):1881-1883. doi:10.1080/0300799 5.2018 .1510830
22. Tang N, Li D, Wang X, et al. Abnormal coagulation parameters are associated with poor prognosis in patients with novel coronavirus pneumonia. J Thromb Haemost. 2020;18(4):844-847. doi:10.1111/ jth. 14768

23. Vervloet MG, Thijs LG, Hack CE. Derangements of coagulation and fibrinolysis in critically ill patients with sepsis and septic shock. Semin Thromb Hemost. 1998;24(1):33-44. doi:10.1055/s-2007995821

24. Iba T, Levy JH, Connors JM, et al. The unique characteristics of COVID-19 coagulopathy. Crit Care. 2020;24(1):360. doi:10.1186/ s13054-020-03077-0

25. Levi M, Thachil J, Iba T, et al. Coagulation abnormalities and thrombosis in patients with COVID-19. Lancet Haematol. 2020;7 (6):e438-e440. doi:10.1016/S2352-3026(20)30145-9

26. Mehta P, McAuley DF, Brown M, et al. COVID-19: consider cytokine storm syndromes and immunosuppression. Lancet. 2020;395 (10229):1033-1034. doi:10.1016/S0140-6736(20)30628-0

27. Tsujimoto S, Mokuda S, Matoba K, et al. The prevalence of endoscopic gastric mucosal damage in patients with rheumatoid arthritis. PLoS One. 2018;13(7):e0200023. doi:10.1371/journal. pone. 0200023
Infection and Drug Resistance

\section{Publish your work in this journal}

Infection and Drug Resistance is an international, peer-reviewed openaccess journal that focuses on the optimal treatment of infection (bacterial, fungal and viral) and the development and institution of preventive strategies to minimize the development and spread of resistance. The journal is specifically concerned with the epidemiology of

\section{Dovepress}

antibiotic resistance and the mechanisms of resistance development and diffusion in both hospitals and the community. The manuscript management system is completely online and includes a very quick and fair peerreview system, which is all easy to use. Visit http://www.dovepress.com/ testimonials.php to read real quotes from published authors. 